\title{
Performance evaluation of a TOA-based trilateration method to locate terminals in WLAN
}

\author{
Fernán Izquierdo, Marc Ciurana, Francisco Barceló, Josep Paradells and Enrica Zola
}

\begin{abstract}
Nowadays, several systems are available for outdoor localization, such as GPS, assisted GPS and other systems working on cellular networks. However, there is no proper location system for indoor scenarios. Research into designing location systems for 802.11 networks is being carried out, so locating mobile devices on global networks (GSM/cellular + GPS + WLAN) finally seems feasible. The technique presented in this paper uses existing wireless LAN infrastructure with minor changes to provide an accurate estimation of the location of mobile devices in indoor environments. This technique is based on round-trip time (RTT) measurements, which are used to estimate distances between the device to be located and WLAN access points. Each RTT measurement estimates the time elapsed between the RTS (Request-to-Send) and the CTS (Clear-to-Send) frame of the 802.11 standard. By applying trilateration algorithms, an accurate estimation of the mobile position is calculated.
\end{abstract}

Index Terms - positioning, ranging, round-trip time, RTSCTS mechanism, time of arrival, triangulation, WLAN.

\section{INTRODUCTION}

Some of the most important new and upcoming mobile services are location-based services and applications which allow mobile users to access services based on their geographic position. These services and applications include emergency rescue, resource tracking and management, tourist information, location-sensitive billing, points of interest, games, and so on. Systems specifically oriented toward positioning and navigation (i.e. GPS) and systems which operate over cellular networks like GSM already exist; however, to date the solutions proposed for indoor environments (e.g. Cell-ID methods for GSM) have not been not particularly accurate.

This paper presents a new indoor WLAN location technique based on distance measurements provided by time-of-arrival (TOA) estimations - which are in turn based on round-trip

Manuscript received on 19 October 2005. This research has been funded by the Spanish Government through the MECD and ERDF project TIC 200301748, and by the EU under the Sixth Integrated FP IST LIAISON Project.

M. Ciurana is a researcher at the Data Networks Engineering Department at the Technical University of Catalonia, Spain (tel.: 0034 934017808, e-mail: mciurana@entel.upc.edu).

F. Izquierdo, F. Barceló, J. Paradells, and E. Zola are researchers at the Data Networks Engineering Department at the Technical University of Catalonia, Spain (e-mail: \{fernani,barcelo,teljpa,enrica\}@entel.upc.edu). time (RTT) measurements - between the device to be located (in this case a laptop PC with a WLAN card) and WLAN access points (APs). An important characteristic of this system is its simplicity [1], as our intention was to use an existing WLAN infrastructure with minor changes to provide an accurate estimation of the position of the mobile device. The system is divided into two subsystems: the ranging subsystem and the positioning subsystem. The former estimates the distances between the mobile device and the APs, and the latter calculates the device's position using the distances already estimated and the APs' known positions.

\section{RANGING SYSTEM}

\section{A. RTT Estimation}

Round-trip time is the time a signal takes to travel from a transmitter to a receiver and back again, in this case from a mobile device to a fixed AP with a known position. The RTT estimation is taken between two consecutive frames under the 802.11 standard: a frame sent by the transmitter and an answering frame from the receiver. The RTS (Request-toSend) and CTS (Clear-to-Send) [3] frames of the 802.11 standard are used. Therefore, the RTT can be measured from the last segment of the RTS frame sent to the first segment of the CTS frame received, as shown in Figure 1.

As the overall (i.e. propagation plus processing) RTT is expected to be in the order of microseconds, it is not possible to estimate it with software. Therefore, the RTT is measured by a hardware subsystem based on the FAST [4] family and connected to the WLAN card and to the parallel port in the mobile device (see Figure 2). This hardware subsystem starts counting using the built-in $44 \mathrm{Mhz}$ clock from the WLAN card when it detects the end of an RTS frame in the WLAN card, and it stops counting when the corresponding CTS frame arrives. Then, using the parallel port, it sends its value, slotted in $44 \mathrm{Mhz}$ periods, to the laptop PC, which is continuously sending pings to the APs and storing the RTT values.

It should be possible to estimate a distance using only one $R T T$ measurement. However, the RTT is highly time-variant due to the impact of multipath [5] and clock quantification errors [6]. The results presented below show that 300 RTT values are needed to accurately estimate a distance, due to the indoor radio channel's characteristics. 


\section{B. Distance Estimation}

First, a set of RTT estimations with zero distance between the mobile device and the AP is obtained (the propagation times $t_{p}$ should be zero), in order to calibrate the time the AP takes to process the query. The value obtained is assumed to be the $t_{\text {proc }}$ RTS part in Figure 1 so that it can be used as an offset for measurements at a non-zero distance. Thus, whatever the distance $(a)$ that separates the transmitter and the receiver, the RTT measured will be greater than at distance zero. Consequently, by applying the offset obtained, it is possible to find the $\triangle R T T$ :

$$
\Delta R T T=R T T_{a}-R T T_{0}
$$

Once the $300 \triangle R T T$ values are calculated, the distance between the transmitter and receiver can be obtained by

$$
d=c \cdot t_{p}=(c \cdot \Delta R T T) / 2 .
$$

However, in accordance with the special characteristics of the indoor radio channel, special algorithms should be used to diminish the multipath and LOS/NLOS (Line-of-Sight/NonLine-of-Sight) problems which lead to higher propagation time measurements. We propose two methods: the first is based on the delay propagation characteristics of the signals, and the second, more empirical method is based on tables made using a set of measurements taken. Both methods were tested in real working environments.

\section{1) Propagation-Based Method}

In this method, the distance is calculated using the average $R T T$ value ( $\eta$, measured in number of clock cycles) obtained from all the measurements, as this value provides the best distance estimation out of all the possible alternatives. Other alternatives, such as using the half range RTT value, the RTT mode, the average of $n$ minimum $R T T$ values and $\eta$ - $n$ times the standard deviation as an estimator were also tested but are not reported in this paper (see [7] for details). With this estimator, taking into account the fact that a $44 \mathrm{Mhz}$ clock was used and that RTT is the return time, Equation (2) can be rewritten as

$$
d=\left(\left(\eta_{\mathrm{a}}-\eta_{0}\right) \cdot 3 \cdot 10^{8}\right) /\left(2 \cdot 44 \cdot 10^{6}\right) .
$$

During the development process, it was observed that all the distances estimated were greater than the real distance; therefore, the estimated distance had to be divided by an empirical coefficient to correct the estimated value. The empirical coefficient is justified by the special characteristics of the multipath [8] indoor radio propagation channel, which can increase the theoretical RTT expected. Only one coefficient was used regardless of whether the system was working in an LOS or NLOS situation. In theory, NLOS cases need a higher coefficient than LOS cases due to the increase in the delay spread, but real measurements have shown us that there is no real need for two empirical coefficients.

The empirical value used was $k=1.32$, the average value of the excess errors obtained. Taking into account this empirical coefficient, the formula for calculating the distance as follows:

$$
d=\left(\left(\eta_{\mathrm{a}}-\eta_{0}\right) \cdot 3 \cdot 10^{8}\right) /\left(2 \cdot 44 \cdot 10^{6} \cdot k\right) .
$$

\section{2) Empirical Table Method}

This method does not use a propagation formula to estimate the distance: it uses the average $R T T$ value to empirically create a table which associates the $R T T$ values with a distance range, so different regions centered on the mobile device are created depending on the RTT value obtained. This method could also be seen as a basic fingerprinting method, as it prestores RTT information and tries to match the new RTT measured with the pre-stored RTT and its corresponding region. However, it should also be noted that this does not provide an exact distance, as a region is presented instead.

\section{Evaluation of the Methods}

The two methods were evaluated by taking several sets of $R T T$ measurements, in order to finally select one of them for the RTT estimation in the ranging system. The results show that, using the propagation-based method and the average $R T T$ values, the measurement errors varied from an average of 2.82 $\mathrm{m}$ when empirical coefficients were not used to $0.64 \mathrm{~m}$ when they were. Moreover, the empirical table method can achieve an accuracy of less than $2 \mathrm{~m}$ in $56.66 \%$ of cases. Therefore, the accuracy provided by the propagation-based method is greater. Furthermore, this method responds better when the working conditions vary, as a small change in the empirical coefficient may suffice in adapting to new conditions, but in the tables method the entire table must be rearranged.

\section{Experimental Test Bed}

The experimental test bed consists of several distance estimations taken with the ranging system in the research laboratory and its surroundings, under different conditions and with varying numbers of people in the laboratory or in other rooms, at different times of the day, at various temperatures, and under different weather conditions. Therefore, all the measurements were taken in a real indoor working environment and without differentiating between LOS and NLOS situations. The access point in a Linksys Wireless-G Broadband Router ${ }^{\circledR}$ was used in all cases.

\section{1) Measurements and Performance}

Two sets of measurements were taken. In the first set, the accuracy of the ranging system was studied by performing several range estimations at different distances. Table 1 shows the absolute and relative errors obtained for every distance. In the second set of measurements, the general probability distribution of the distances estimated by the ranging system was obtained. (The use of this probability distribution by the positioning module to simulate the position estimation results will be discussed later on in this paper, in Section III.C.) The second set of measurements consists of 450 distance estimations $(450 * 300$ RTT measurements), measured at a constant distance of $10 \mathrm{~m}$, after the initial calibration at $0 \mathrm{~m}$.

The 450 distance estimations were fed into Matlab ${ }$, each of which was assigned to the number of times it occurred. This 
was to create a probability distribution of the distance measured. Ideally, all the distances measured should be $10 \mathrm{~m}$; however, due the multipath indoor radio channel [8], the ranging system obtains distances from $8.80 \mathrm{~m}$ to $12.80 \mathrm{~m}$. Therefore, Matlab was also used to fit this empirical histogram to a known probability distribution. The best fit was found to be a Gaussian distribution, as can be seen in Figure 3 .

\section{Positioning System}

\section{A. Introduction}

As stated above, the mobile device's position is determined once the distance estimations from a set of access points are known. This can be done with triangulation algorithms and the distance measurements of at least three access points at a known location (Figure 4).

The solution technique for this problem begins by finding the intersection of several circumferences, as the technique proposed in this paper is designed for 2D. The point of intersection of these circumferences is obtained by solving the $n$ nonlinear equations simultaneously to eliminate two coordinates, where $n$ is the number of APs. Due to the nonlinearity of these equations, if a solution of $n$ - 1 dimensions has to be found, $n$ equations are required; that is why in this case of $2 \mathrm{D}$ positioning, at least three access points are needed to determine each position. However, solving a system of $n$ nonlinear equations simultaneously is not feasible because this results in a high-degree nonlinear equation [9], which makes calculating the mobile device's position more complex.

Linearizing the system of equations geometrically turns the problem into one of finding the point of intersection of several planes. Since approximate distances are used, the solution of the linear system is not completely determined. Consequently, the criterion of minimizing the mean square error between the estimated position (the position which will be obtained in this system) and the real position has to be determined using specific algorithms if good results are to be obtained.

\section{B. Positioning Algorithms}

\section{1) Linear Least Squares}

Although this algorithm is not very accurate, it provides an initial position which can be used afterwards by other positioning algorithms (i.e. Nonlinear Least Squares and Independent Time GPS Least Squares) as the initialization value for their iterations.

First, the system of equations mentioned above must be linearized. In order to do so, the $j$ th constraint is used as a linearizing tool [9]. Adding and subtracting $x_{j}$ and $y_{j}$ in the typical circumference equation gives

$$
\left(x-x_{j}+x_{j}-x_{i}\right)^{2}+\left(y-y_{j}+y_{j}-y_{i}\right)^{2}=r_{i}^{2} \text {. }
$$

with $(i=1,2, \ldots, j-1, j+1, \ldots \mathrm{n})$. Expanding and regrouping the terms and selecting the first constraint $(j=1)$ leads to a linear system of ( $n-1)$ equations with two unknowns:

$$
\begin{aligned}
& d_{i j}=\sqrt{\left(x_{i}-x_{j}\right)^{2}+\left(y_{i}-y_{j}\right)^{2}} \\
& \left(x-x_{1}\right) \cdot\left(x_{2}-x_{1}\right)+\left(y-y_{1}\right) \cdot\left(y_{2}-y_{1}\right)=\frac{1}{2}\left[r_{1}^{2}-r_{2}^{2}+d_{21}^{2}\right]=b_{21} \\
& \left(x-x_{1}\right) \cdot\left(x_{3}-x_{1}\right)+\left(y-y_{1}\right) \cdot\left(y_{3}-y_{1}\right)=\frac{1}{2}\left[r_{1}^{2}-r_{3}^{2}+d_{31}^{2}\right]=b_{31} \\
& \vdots \\
& \left(x-x_{1}\right) \cdot\left(x_{n}-x_{1}\right)+\left(y-y_{1}\right) \cdot\left(y_{n}-y_{1}\right)=\frac{1}{2}\left[r_{1}^{2}-r_{n}^{2}+d_{n 1}^{2}\right]=b_{n 1} .
\end{aligned}
$$

This linear system can be simplified into a matrix form:

$$
A \vec{x}=\vec{b}
$$

where

$$
\begin{aligned}
A & =\left(\begin{array}{cc}
x_{2}-x_{1} & y_{2}-y_{1} \\
x_{3}-x_{1} & y_{3}-y_{1} \\
\cdots & \ldots \\
x_{n}-x_{1} & y_{n}-y_{1}
\end{array}\right) \\
\vec{x} & =\left(\begin{array}{c}
x-x_{1} \\
y-y_{1}
\end{array}\right) \\
\vec{b} & =\left(\begin{array}{l}
b_{21} \\
b_{31} \\
\ldots \\
b_{n 1}
\end{array}\right) .
\end{aligned}
$$

Once the system has been linearized, and since the distances $r i$ are only approximate, the problem requires the determination of $\vec{x}$ such that $A \vec{x} \approx \vec{b}$ :

$$
A^{T} A \vec{x}=A^{T} \vec{b}
$$

There are several methods for solving this equation. Since it is assumed that the access points will be logically placed (not in a row or colinearly), this means that $A^{T} A$ is non-singular and well conditioned. Therefore, the method used is the following:

$$
\vec{x}=\left(A^{T} A\right)^{-1} A^{T} \vec{b}
$$

\section{2) Nonlinear Least Squares (Newton)}

The nonlinear least squares algorithm uses an initial estimation of the position, which has already been obtained by the linear LS, and minimizes the sum of the squares of the errors in the distances. Minimizing the sum of the square errors is a fairly common problem in applied mathematics for which various algorithms are available. The Newton iteration was selected to find the 'optimal' solution $\mathrm{P}(x, y)$. Introducing $f$, $g$ and the Jacobian matrix $J$, and applying the Newton iteration, gives

$$
\vec{R}_{k+1}=\vec{R}_{k}-\left(J_{k}^{T} J_{k}\right)^{-1} J_{k}^{T} \vec{f}_{k}
$$


where $\vec{R}_{k}$ denotes the $k t h$ approximate solution. The subscript $k$ in $\mathrm{J}$ and $\mathrm{f}$ means that these quantities are evaluated at $\vec{R}_{k}$. Obviously, $\vec{R}_{1}=(\tilde{x}, \tilde{y})^{T}$. Finally, using the explicit form of the function $f_{i}(x, y)$ gives

$$
\begin{aligned}
& J^{T} J=\left(\begin{array}{cc}
\sum_{i=1}^{n} \frac{\left(x-x_{i}\right)^{2}}{\left(f_{i}+r_{i}\right)^{2}} & \sum_{i=1}^{n} \frac{\left(x-x_{i}\right)\left(y-y_{i}\right)}{\left(f_{i}+r_{i}\right)^{2}} \\
\sum_{i=1}^{n} \frac{\left(x-x_{i}\right)\left(y-y_{i}\right)}{\left(f_{i}+r_{i}\right)^{2}} & \sum_{i=1}^{n} \frac{\left(y-y_{i}\right)^{2}}{\left(f_{i}+r_{i}\right)^{2}}
\end{array}\right) . \\
& J^{T} \vec{f}=\left(\begin{array}{l}
\sum_{i=1}^{n} \frac{\left(x-x_{i}\right) f_{i}}{\left(f_{i}+r_{i}\right)} \\
\sum_{i=1}^{n} \frac{\left(y-y_{i}\right) f_{i}}{\left(f_{i}+r_{i}\right)}
\end{array}\right) .
\end{aligned}
$$

Using these matrices and equations, the algorithm performs iterations until the difference from the previous iteration and the next one is smaller than $\delta$, which is a completely modifiable threshold.

\section{3) Independent Time GPS Least Squares}

This is the basic algorithm that forms the mathematical basis of a GPS [10] system. It is used to solve the navigation equations before introducing the Kalman filter concept. "Independent time" stands for the independency between the next position and the positions calculated at previous times, while the Kalman filter would include preceding position information in the calculation of the present position. This algorithm only takes into account the estimated distances between the device to be located and the APs and the positions of these APs.

The algorithm needs an initial, approximate position of the device to be located. The observable for each AP is the prefit residual, which is the difference between the measured and the modeled estimation of the distance. In our case, the modeled distance corresponds to the distance between the access point and the initial estimated position. The solution to these equations (navigation equations in terms of GPS) is the correction that must be applied to the initial position:

$$
P_{j}+c \cdot d t_{j}-\delta_{j} \simeq \sqrt{\left(x-x_{j}\right)^{2}+\left(y-y_{j}\right)^{2}+\left(z-z_{j}\right)^{2}}+c \cdot d t .
$$

where $j=1,2, \ldots, n(n \geq 4)$. Every equation is linearized using the Taylor approximation around the initial position, in order to apply the least squares method to solve the equation system. Applying this method yields

$$
\left[\begin{array}{c}
P_{1}-\rho_{1,0}+c \cdot d t_{1}-\delta_{1} \\
\vdots \\
P_{n}-\rho_{n, 0}+c \cdot d t_{n}-\delta_{n}
\end{array}\right]=\left(\begin{array}{cccc}
\frac{x_{0}-x_{1}}{\rho_{1,0}} & \frac{y_{0}-y_{1}}{\rho_{1,0}} & \frac{z_{0}-z_{1}}{\rho_{1,0}} & 1 \\
\vdots & \vdots & \vdots & \vdots \\
\frac{x_{0}-x_{n}}{\rho_{n, 0}} & \frac{y_{0}-y_{n}}{\rho_{n, 0}} & \frac{z_{0}-z_{n}}{\rho_{n, 0}} & 1
\end{array}\right)\left[\begin{array}{c}
d x \\
d y \\
d z \\
c \cdot d t
\end{array}\right] .
$$

The algorithm is applied iteratively for better results. It reconstructs the navigation equations by using the position obtained and recalculating the new iteration position, until the difference between the results of one iteration and those of the next is smaller than $\delta$, which once again is a completely modifiable threshold.

\section{Experimental Test Bed \\ 1) Simulations}

In order to evaluate the accuracy of the two triangulation algorithms, they were implemented using Matlab. Several simulations were performed, each carried out as follows:

- The positions of the three access points were introduced, as well as the position of the mobile device which was going to be estimated.

- The simulation program calculated the exact distances from each AP to the mobile device.

- These distances were modified using the resulting probability distribution of the distance estimated, i.e. the exact distance from the mobile device to AP A was $10 \mathrm{~m}$. Instead of using these $10 \mathrm{~m}$ distances, the simulation used the Gaussian probability distribution obtained from the measurements of the ranging system for $10 \mathrm{~m}$, as presented in Section II.D.1. This probability distribution was divided into slots of $10 \mathrm{~cm}$. Therefore, there was a probability associated with each possible distance the ranging system could measure. This is shown in Figure 5, in which there are three access points placed at 4, 12, and $17 \mathrm{~m}$ respectively, but these distances were replaced by their corresponding Gaussian bells. The same probability distribution was used for all distances because previous results show that there are no major variations when different distances are involved.

- The simulation found the estimated position of the mobile device using the aforementioned algorithms for each of the possible distances estimated at each of the three access points. This means that each AP probability distribution was used at all possible points and that they were combined with the remaining APs to find all the possible position estimations and the probability associated with each of them. Once these estimations were known, they were subtracted from the mobile device's real position to find the error. This process was carried out using loops which combined every slot at each access point with all the possible slots in the rest of the access points, as shown in Figure 6. 
- Finally, the cumulative probability function of the position estimation error for every positioning algorithm was found.

Since the results depended on the relative distance between the mobile and the three access points, the simulations were performed taking various scenarios into consideration. In all these scenarios, the geometric dilution of precision was estimated, and, since APs are assumed to be rationally placed, average GDOP values were used.

As can be seen in Figure 7, when APs are rationally placed (non-colinearly, for instance) the system can be $90 \%$ accurate between $2 \mathrm{~m}$ and $2.3 \mathrm{~m}$. It can be also seen that the Nonlinear Least Squares (Newton) algorithm outperforms the GPS Least Squares algorithm.

\section{CONCLUSIONS AND FUTURE WORK}

The main objective of this paper was to study a new WLAN location technique, which uses the existing WLAN infrastructure with minor changes, in order to provide an accurate estimation of the location of mobile devices indoors. This technique is based specifically on RTT measurements from frames ranging from the 802.11 RTS frame to the 802.11 CTS frame. The RTT measurements were used to estimate the distances from at least three access points to the mobile device; once these distances were calculated the system performed triangulation methods to find the mobile device's position. The results obtained were satisfactory: $90 \%$ accuracy within the range of 2 to $2.3 \mathrm{~m}$, using only three access points. Moreover, only minor changes in the existing WLAN infrastructure were needed; a simple hardware circuit, which could be directly implemented in future WLAN card designs by manufacturers at a low cost, is all that is required.

These future lines of work could improve the results of the technique:

- Research to find out the impact on accuracy of an improvement in the clock's frequency.

- Research on the self-learning process, namely the ability to estimate the coefficient depending on the environment (multipath radio channel) and other factors related to the traffic load of the network, such as the APs' processing time. This might be a way to avoid having to carry out the calibration process and the empirical and manual calculation of the coefficient.

- Research on the use of specific algorithms to detect whether the system is working in LOS or NLOS conditions in an attempt to mitigate the multipath problems by using specific coefficients.

\section{ACKNOWLEDGMENTS}

The authors wish to thank Alfonso Mendez, Vicente Ruiz at the Electronic Engineering Department at UPC, and J. Manuel Berbel at the Data Networks Engineering Department at UPC for their help in assembling the hardware module.

\section{REFERENCES}

[1] Moustafa Youssef, "Horus: A WLAN-Based Indoor Location Determination System", Department of Computer Science, University of Maryland, 2004.

[2] Xinrong Li; Pahlavan, K.; Latva-aho, M.; Ylianttila, M., "Comparison of iIndoor Geolocation Methods in DSSS and OFDM Wireless LAN Systems", IEEE VTS-Fall VTC 2000. 52nd, Volume 6, 24-28 Sept. 2000, pp. 3015-3020.

[3] McCrady, D.D.; Doyle, L.; Forstrom, H.; Dempsey, T.; Martorana, M.; "Mobile Ranging Using Low-accuracy Clocks", IEEE Transactions on Microwave Theory and Techniques, Volume 48, Issue 6, June 2000, pp.951-958.

[4] Philips Semiconductors, Information and Datasheets. Available online at http://www.standardproducts.philips.com.

[5] Andrew M. Ladd, Kostas E. Bekris, Algis P. Rudys, Dan S. Wallach, and Lydia E. Kavraki, "On the Feasibility of Using Wireless Ethernet for Indoor Localization", IEEE Transactions On Robotics And Automation, Vol. 20, No. 3, pp.555-559, June 2004.

[6] André Günther, Christian Hoene: Measuring Round Trip Times to Determine the Distance Between WLAN Nodes. Networking 2005, pp. 768-779.

[7] Fernán Izquierdo, "Wireless LAN Location Technique based on RoundTrip-Time Measurements" Thesis, 2005, ETSETB, UPC.

[8] Homayoun Hashemi, "The iIndoor Radio Propagation Channel", Proceedings of the IEEE, Vol. 81, No.7, July 1993, pp. 943-968.

[9] W. Murphy and W. Hereman, "Determination of a Position in Three Dimensions Using Trilateration and Approximate Distances", 1995, tech. report MCS-95-07, Colorado School of Mines, Golden, CO.

[10] Gilbert Strang, "The Mathematics of GPS", SIAM News, Volume 30, Number 5, June 1997 (C) 1997, Society for Industrial and Applied Mathematics.

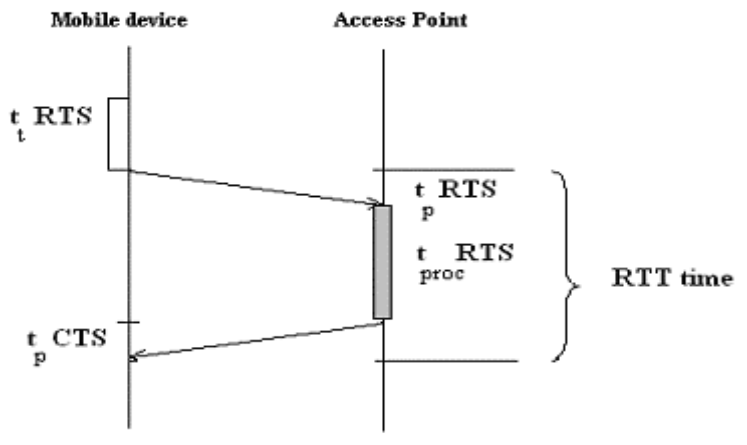

Figure 1. RTT measurement using RTS/CTS frames

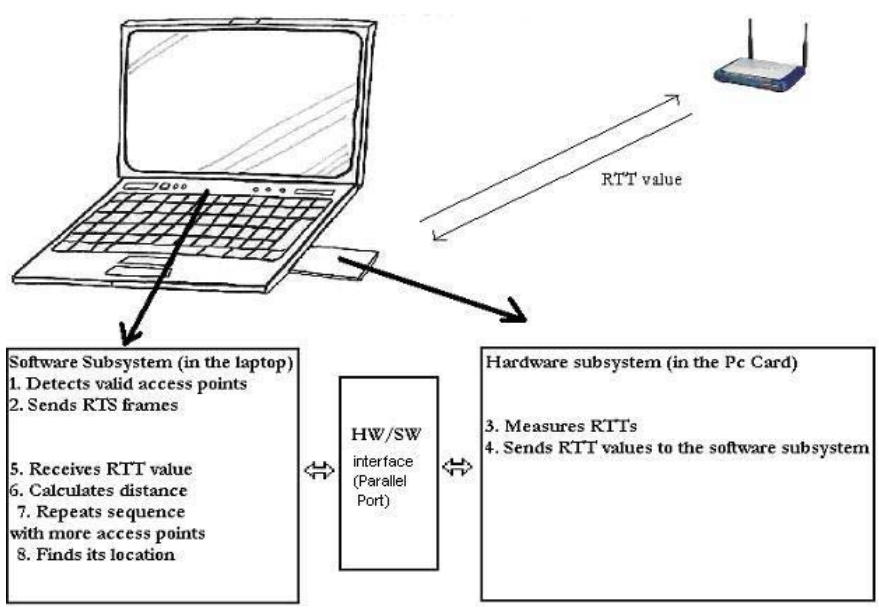

Figure 2. Block diagram of the ranging system 


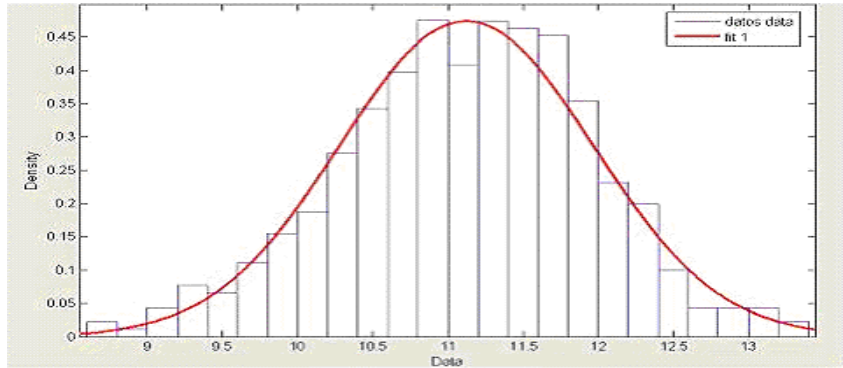

Figure 3. Histogram of distance measurements

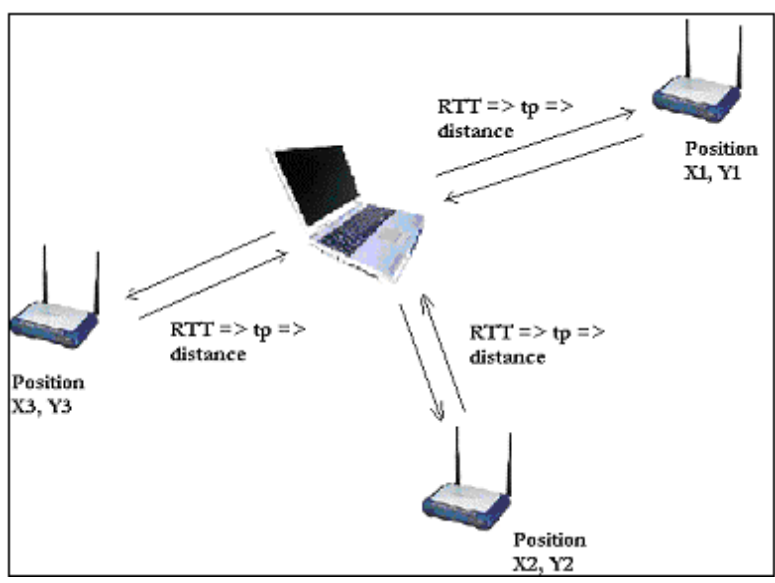

Figure 4. RTT-based triangulation

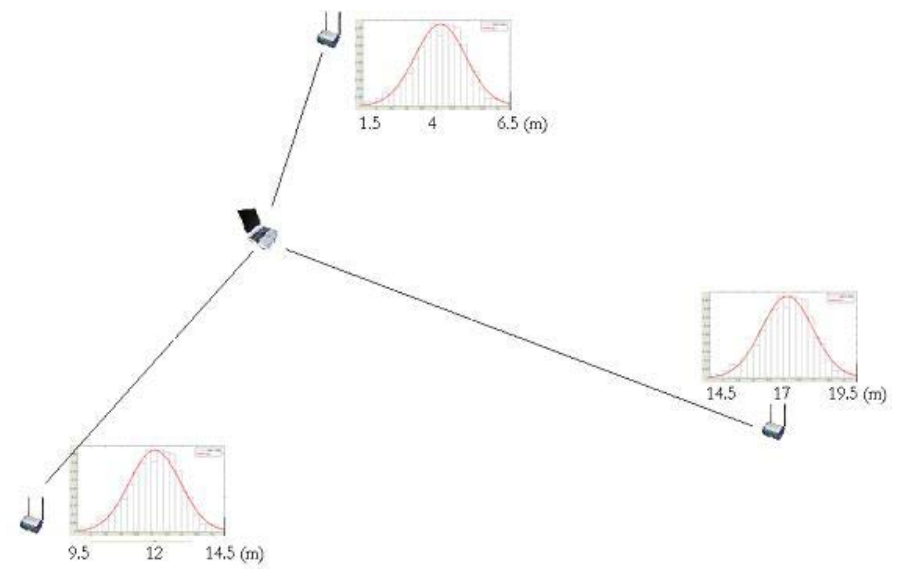

Figure 5. Simulation of the triangulation (I)

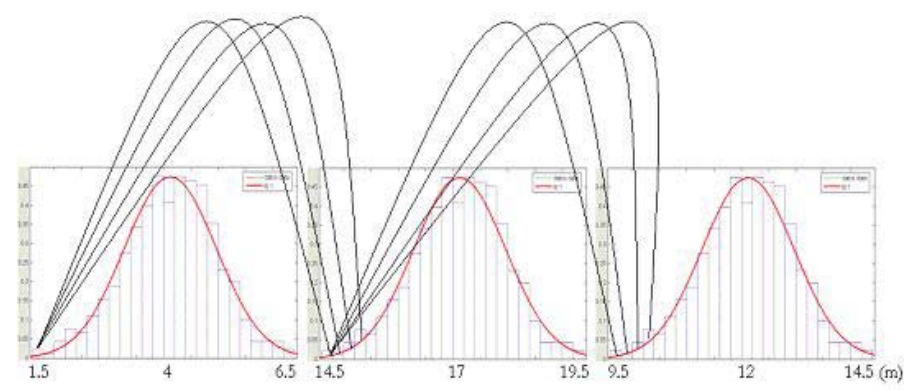

Figure 6. Simulation of the triangulation (II)

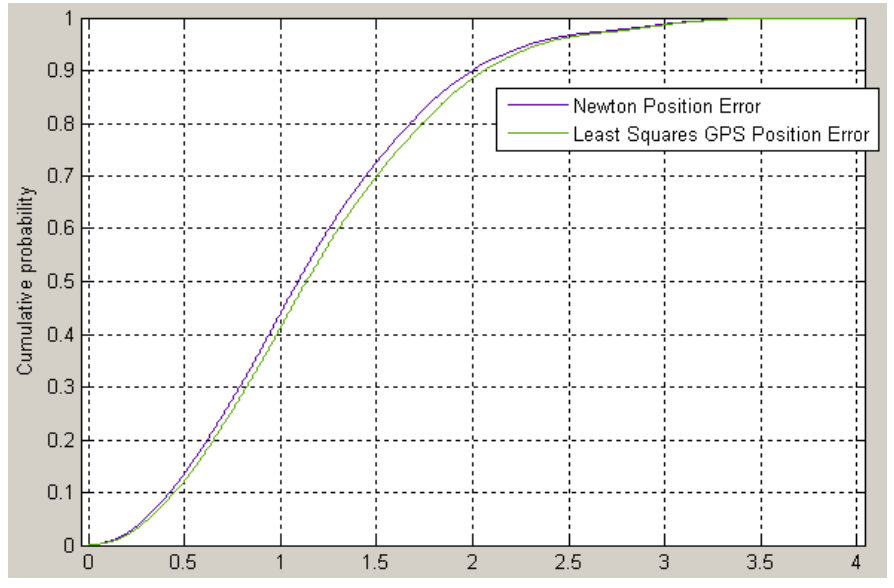

Figure 7. CDFs' positioning error (a)

Table I. RESULTS OF THE RANGING SYSTEM

\begin{tabular}{|l|c|l|l|l|}
\hline Distances & \multicolumn{1}{|c|}{$\mathbf{~ m}$} & $\mathbf{1 0} \mathbf{~ m}$ & $\mathbf{1 5} \mathbf{~ m}$ & $\mathbf{2 0} \mathbf{~ m}$ \\
\hline Average & $0.51 \mathrm{~m}$ & $0.51 \mathrm{~m}$ & $1.38 \mathrm{~m}$ & $0.47 \mathrm{~m}$ \\
error & $(10.2 \%)$ & $(5.1 \%)$ & $(9.2 \%)$ & $(2.3 \%)$ \\
\hline Maximum & $1.21 \mathrm{~m}$ & $1.24 \mathrm{~m}$ & $2.88 \mathrm{~m}$ & $1.01 \mathrm{~m}$ \\
error & $(24.2 \%)$ & $(12.4 \%)$ & $(19.2 \%)$ & $(5.0 \%)$ \\
\hline
\end{tabular}

